\title{
Refactoring for Performance with Semantic Patching: Case Study with Recipes
}

\author{
Michele Martone $^{\star 1[0000-0003-3239-8554]}$ and Julia Lawall ${ }^{2}$ \\ 1 Leibniz Supercomputing Centre, Garching bei Muenchen, Germany \\ Michele.Martone@lrz.de \\ 2 Inria-Paris, Paris, France \\ Julia.Lawall@inria.fr
}

\begin{abstract}
Development of an HPC simulation code may take years of a domain scientists' work. Over that timespan, the computing landscape evolves, efficient programming best practices change, APIs of performance libraries change, etc. A moment then comes when the entire codebase requires a thorough performance lift. In the luckiest case, the required intervention is limited to a few hot loops. In practice, much more is needed. This paper describes an activity of programmatic refactoring of $\approx 200 \mathrm{k}$ lines of $\mathrm{C}$ code by means of source-to-source translation. The context is that of a so-called high level support provided to the domain scientist community by a HPC service center. The motivation of this short paper is the immediate reuse potential of these techniques.
\end{abstract}

\section{Introduction}

GADGET3 (from now on, GADGET) is a code simulating large-scale structure (galaxies and clusters) formation. Not counting forks, it has a codebase of around $200 \mathrm{kLOC}$ (thousands of Lines of Code) in C. It is being developed by a geographically sparse community of domain scientists (astrophysicists). Code revisions progress using a revision control system.

GADGET revolves around the concept of particle. A particle and its associated quantities are modeled using a few $\mathrm{C}$ structs, e.g.: struct $\mathrm{P}$ \{float $\mathrm{x}, \mathrm{y}, \mathrm{z} ;\} * \mathrm{p} ;$. Here three quantities are shown; GADGET has around a hundred of them, enabled/disabled via several \#ifdefs. Memory use is dominated by a few dynamically allocated global arrays of similar structs. The number crunching defining most of the code accesses these arrays using expressions similar to $\mathrm{p}[\mathrm{e}] . \mathrm{x}$ (e being an indexing expression). This data layout goes by the name of Arrays of Structures (AoS). The GADGET community finds this layout very handy: the AoS definitions reside in one single header maintained by the project lead. Collaborators maintain separate source files with functionality (in jargon, physics modules) de-/activated by \#ifdefs.

While appreciated by the community, AoS performs suboptimally on the frequently occurring streaming access loops. On current architectures, the complementary layout - Structures of Arrays (SoA) - performs better here, for

\footnotetext{
* Corresponding author.
} 
it favours compiler autovectorization [5]. The SoA equivalent of the previously sketched AoS is: struct $\mathrm{P}\{$ float $* \mathrm{x}, * \mathrm{y}, * \mathrm{z} ;\} \mathrm{p}$; , with dynamically allocated arrays $\mathrm{p} . \mathrm{x}, \mathrm{p} . \mathrm{y}$, and $\mathrm{p} . \mathrm{z}$. Besides redefinition of particle structs and introducing a few new variables, porting AoS to SoA requires rewriting the bulk of the code. Namely, translating all the non-trivial expressions containing combinations

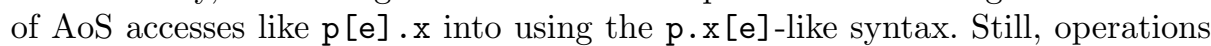
that move particles are more practical with AoS, than on structures scattered across a few hundred arrays. Thus, one may want to retain the AoS definitions, and use scatter/gather operations to occasionally convert to/from SoA, like in sorting or load balancing across distributed processes over MPI.

GADGET is a major astrophysical code and improving its performance is highly desirable. Baruffa et al. 2] estimated a performance improvement with SoA as exceeding $2 \times$, but their study was limited to an excerpt of circa $1 \mathrm{kLOC}$. The present article tackles the problem of backporting SoA to the entire $\approx 200$ kLOC code, respecting its many build-time \#ifdef variants.

The present work has been carried within a longer-term (so-called high level support) activity at LRZ, and has targeted both a legacy version (P-GADGET3) and a development one (codenamed OpenGadgeT3, from non-public source repositories) forks, both from the Max-Planck Institute for Astrophysics in Garching, Germany. These ( 7,6 , 6]) are derivatives of GADGET2 [8]

This article applies to both and addresses either by the namesake GADGET. Although we are aware of $\mathrm{C}++$-based techniques to use a SoA semantics with AoS syntax, we had to rule those out: keeping the code in $\mathrm{C}$ has been a project requirement.

\section{Prospective Factorization Steps and Tool Choice}

Essential to any AoS to SoA translation are: 1. Identifying AoS members to be made into SoA arrays; 2. Declaring new SoA types and variables in a global scope; 3. For each new SoA array, adding de-/allocation and AoS $\leftrightarrow$ SoA gather/scatter primitives; 4. Accessing each new SoA array by mutating corresponding AoS expressions. Such changes are constrained by interrelated factors:

- timeliness: How to quickly change so many lines of code?

- correctness: How to avoid introducing mistakes? Note the aggravation of having numerous build-time code paths implied by the \#ifdefs.

- flexibility: Can we enact only a partial SoA translation, possibly on demand?

- continuity: Can we develop in AoS, transforming only before build and run?

- acceptance: How to have the community accept the proposed solution?

Timeliness requires an automated tool. Correctness calls for a tool having a model of a $\mathrm{C}$ program. A programmable, or at least parametric solution in choosing AoS quantities would be best: we do not know which subset of a complete SoA transition is most performant under which configuration (moreover, several code forks exist). Continuity by preserving the current AoS development culture would maximize acceptance. 
Existing Integrated Development Environments (IDEs) are too primitive for these changes. Regular expressions are tempting but brittle: $\mathrm{C}$ expressions may span multiple lines, and do not generally fit regular expressions. ROSE is a compiler-based infrastructure for developing program analyses and transformations. It requires working at the AST level and regenerates the affected code. Manipulating ASTs can be awkward for the developer, who is more familiar with the code base in its source code representation. The regenerated code does not follow the whitespace usage of the original code, which can lead to many superfluous diffs between the old and new versions. This is especially problematic in the case of a legacy code base, where users are mostly concerned with stability and any changes have to be checked carefully.

These factors suggested the use of the Coccinelle rule-based language for transforming $\mathrm{C}$ code $3 \sqrt{4}$. CocCINELLE is a metalanguage routinely used by the Linux kernel developer community for large-scale updating of API usages and data structure layouts. The use of CoccinelLE on driver code results in mostly short diffs, which is not the case here. Its use in HPC is in its inception.

\section{Thinking out Coccinelle Transformation Rules}

CocCinelLe's rule syntax is a variant of the familiar patch syntax, thus also serving as documentation of the performed changes. A rule may match code at a function, definition, or declaration level, etc. Matching is independent of code layout, sensitive to control-flow, and takes into account type information. The modification specification is intermingled in the rule: that motivates terming this technology semantic patching. Rules can be chained; they involve notions of existential and universal quantifiers, as often found in logic programming languages.

Sec. 2 has enumerated well-defined factorization steps. Below, we describe their counterpart as real-life semantic patches.

\subsection{Identify AoS Variables for Reuse in SoA}

GADGET AoS variables involve variables of type particle data and sph parti- 1 @prtcl_stre cle_data, and their members. The vari- sph_particle_data\}; ables are found by the COCCINELLE rule 3 field list $f$; prtcl_str, at right. Such a rule has two 5 declaration d;

parts. Lines 1-7 describe a set of metavari- 7 e@

ables, which match any term of the indi- 8 (

9 struct id $\{\mathrm{fs}\} * \mathrm{I}$;

cated kind: id (line 2) matches any iden- 10 \&

tifier, limited to a set of possible names $11 \mathrm{ST} \quad\{\mathrm{fs}\} * \mathrm{I}$;

(particle_data and sph_particle_data), 13 d

while I matches any identifier without

restriction. Lines 8-14 provide a pattern that matches a structure declaration. This pattern matches the declaration in three ways, connected as a conjunction by the enclosing ( and ) (lines 8 and 14), and by the occurrences of \& on 
lines 10 and 12. The first pattern (line 9) exposes the details of the declaration, matching id to the name of the structure type, fs to the list of members (referred to by Coccinelle as a field list) and I to the name of the declared variable. The second pattern (line 11) matches ST to the entire type. The third pattern (line 13) matches $\mathrm{d}$ to the complete declaration. A match against a declaration in the $\mathrm{C}$ code only succeeds if all three patterns match the declaration.

Identifying members within the types of the variables matched by the previous rule is done by the following rule. This rule matches members of a previously selected structure (referenced by the redeclaration of id on line 2), such that the members are restricted to have one of a specified list of type $\mathrm{s}$ (line 5). Ellipses ( ... ) (line 7) match the context around each matching member. This rule is applied once for each struct name for which a variable was found by the previous rule. There may furthermore be many matching members. This rule (and those dependent on it) is applied on each possible match.

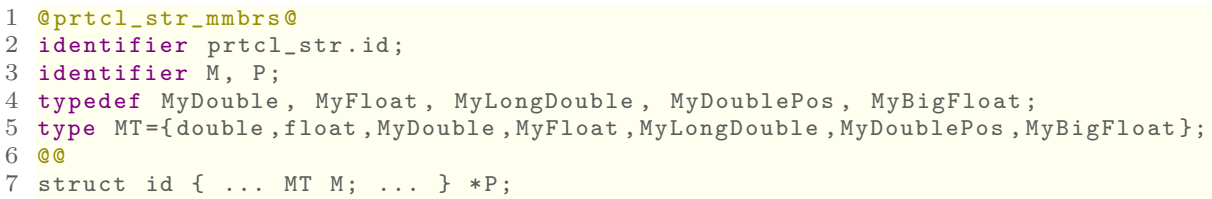

\subsection{Clone Structures and Make them SoA}

Firstly, we want to derive SoA type identifiers id1 from previously matched AoS identifiers id. For this, we exploit the PyтHON scripting functionality. Likewise, we create SoA variable identifiers.

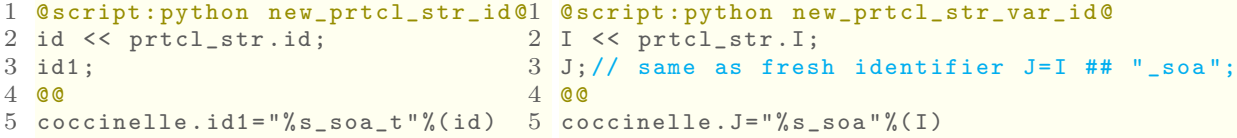

Once the SoA identifiers are ready, we can add the new type definitions in the main header, extern variable declarations in most sources, and the variables themselves in one specific point. The choice of the attach points is crucial here.

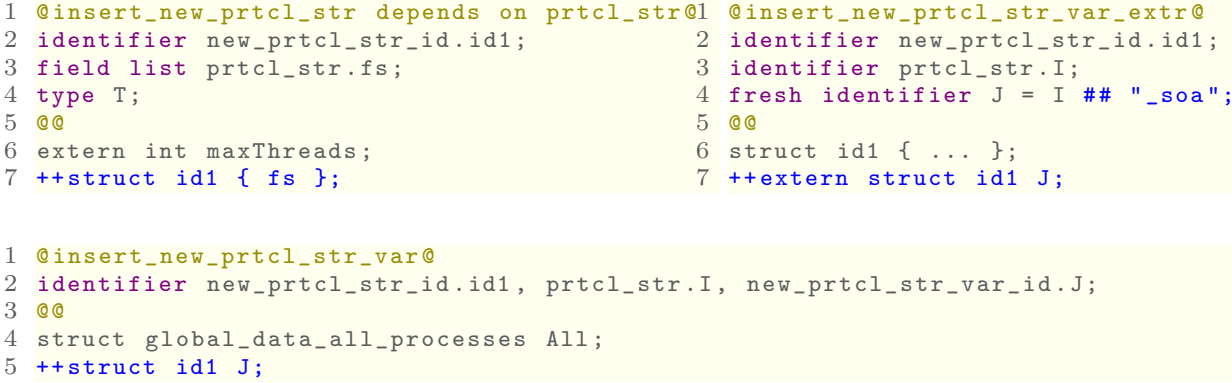


quality of the changes has been ensured by using the internal test suite and a few self-developed custom check scripts. The semantic patches have been developed on extracts of the code, which allowed quick and terse visual inspection of the changes. CoccinelLE preserves existing coding style (e.g. spacing, indentation) very well, which leads to a minimal diff.

Generally, HPC is witnessing a divergence in APIs and programming models. This may be a problem for small, geographically sparse domain scientist teams working on large codebases, risking 1) premature code obsolescence, 2) wasted, repeated effort, and 3) operation of hardware at below-optimal performance. This paper illustrated a novel use of a source-to-source translator in such a context. Adapting these recipes to introduce SoA in another codebase may be straightforward. However, the cleaner a codebase is, the easier it is to develop semantic patches. Adopting coding guidelines and following emerging research software engineering practices [1] will help, no matter how small the team.

\section{References}

1. Anzt, H., Bach, F., Druskat, S., Löffler, F., Loewe, A., Renard, B.Y., Seemann, G., Struck, A., Achhammer, E., Aggarwal, P., et al.: An environment for sustainable research software in Germany and beyond: current state, open challenges, and call for action. F1000Research 9, 295 (Apr 2020). https://doi.org/10.12688/f1000research.23224.1

2. Baruffa, F., Iapichino, L., Hammer, N.J., Karakasis, V.: Performance optimisation of smoothed particle hydrodynamics algorithms for multi/many-core architectures. CoRR abs/1612.06090 (2016), http://arxiv.org/abs/1612.06090

3. Coccinelle: https://archive.softwareheritage.org/swh:1:snp: 207d182d085fcff85a70deb765336ffe63db5c2a; origin=https://github.com/ coccinelle/coccinelle

4. Lawall, J., Muller, G.: Coccinelle: 10 years of automated evolution in the Linux kernel. In: 2018 USENIX Annual Technical Conference, USENIX ATC. pp. 601-614 (2018)

5. Pennycook, S.J., Hughes, C.J., Smelyanskiy, M.: Chapter 8 - optimizing gather/scatter patterns. In: Reinders, J., Jeffers, J. (eds.) High Performance Parallelism Pearls, pp. 143 - 157. Morgan Kaufmann, Boston (2015). https://doi.org/10.1016/B978-012-802118-7.00008-X

6. Ragagnin, A., Dolag, K., Wagner, M., Gheller, C., Roffler, C., Goz, D., Hubber, D., Arth, A.: Gadget3 on GPUs with OpenACC. In: Parallel Computing: Technology Trends, Proceedings of the International Conference on Parallel Computing (ParCo). Advances in Parallel Computing, vol. 36, pp. 209-218. IOS Press (2019). https://doi.org/10.3233/APC200043

7. Ragagnin, A., Tchipev, N., Bader, M., Dolag, K., Hammer, N.: Exploiting the space filling curve ordering of particles in the neighbour search of Gadget3. In: Parallel Computing: On the Road to Exascale, Proceedings of the International Conference on Parallel Computing (ParCo). Advances in Parallel Computing, vol. 27, pp. 411420. IOS Press (2015). https://doi.org/10.3233/978-1-61499-621-7-411

8. Springel, V.: The cosmological simulation code GADGET-2. MNRAS 364, 11051134 (2005). https://doi.org/10.1111/j.1365-2966.2005.09655.x 